\title{
Optimal Balancing of Freeway Traffic Density: Application to the Grenoble South Ring
}

\author{
Dominik Pisarski and Carlos Canudas-de-Wit
}

\begin{abstract}
This paper presents the application of the idea of optimal balancing of traffic density distribution. The idea was previously studied in the papers [1], [2], and here it is implemented to the Grenoble South Ring in the context of the Grenoble Traffic Lab. The traffic on the ring is represented by the Cell Transmission Model that was tuned by using real data and Aimsun micro-simulator. A special attention is paid to the calibration of a flow merging model. A large-scale optimization problem is solved by using decomposition methods and it is implemented by introducing combinatorial procedures. The main difficulties in the implementation as well as the limitations of the designed software are highlighted. Finally, the results of different traffic scenarios on the Grenoble South Ring are presented.
\end{abstract}

\section{INTRODUCTION}

A permanent increase in requirements for transportation motivates intensive studies on control of freeway traffic. A variety of both ramp metering and variable speed limiting methods have been developed and put into practice. The impact of these methods on economics, human living and nature is unquestionable. Shortened delays, reduced pollution, decreased number of accidents are among many other benefits that should be mentioned here.

There are several standard objectives in the optimization of freeway systems. The commonly used is to decrease the time of travel incurred by all drivers while maximizing the total traffic flow passing through a freeway. For this purpose, the objective functions like Total Travel Spent, the Total Travel Distance and the Total Input Volume were introduced. Usually they are combined with some additional terms that penalize abrupt variations in ramp metering and speed limiting signals. For the open-loop optimal control techniques a reader is referred to the papers [3], [4]. Some interesting results on feedback control were proposed for example in [5], [6].

In this paper, we consider the problem of state balancing of the freeway traffic system. The state of such a system is represented by distribution of vehicle density while on-ramp demands are assumed for the inputs. The objective is to find a set of inputs that result in a uniform distribution of density. In practice, this uniform distribution can be seen as an equal inter-distance between vehicles. The equal inter-distance can

Dominik Pisarski is with NeCS team INRIA, Grenoble, France, dominik.pisarski@inria.fr

C. Canudas-de-Wit is a director of reearch at the CNRS, GIPSA-Lab. NeCS team, Grenoble, France, carlos.canudas-de-wit@gipsa-lab.grenobleinp. fr be attractive to the driver's point of view. It reduces the number and intensity of acceleration and deceleration events and therefore, it makes a travel more safety and comfortable while decreasing emission.

The idea of freeway density balancing is intended to be implemented by using feedback control. For that purpose, the crucial is to investigate the structures of equilibria of such a system. The preliminary work was published in the papers [1], [2]. In the first one, the authors addressed the problem of exact balancing. The goal was to find the system input such that the resulting steady state vector is equalized. The analysis on the Cell Transmission Model showed that in order to provide uniformly distributed steady state some condition on the cell parameters must hold. The second paper concerns non-exact balancing i.e. generalization of the previous idea for the cases where a desired exact balanced state does not belong to set of equilibrium points. This paper is to apply and to verify the balancing idea to the model of the Grenoble South Ring.

The optimization is performed on the Cell Transmission representation of the part of the Grenoble South Ring equipped with four control inputs. The model is tuned by using real data and traffic micro-simulator. A special attention is put for merging model calibration. A several traffic scenarios are considered to capture the most interesting aspects of the proposed ideas.

The results presented here have been recently developed in the Grenoble Traffic Lab (GTL) - a real-time traffic data center. The laboratory is intended to collect traffic road infrastructure information as well as to test traffic prediction and control algorithms. It manages a wireless sensor network distributed along the south-ring in Grenoble that allows to measure velocity and flow in a particular position on the road. An important innovation of GTL is that the sensors are placed such to ensure the best conditions for observability ([7], [8]) and controllability. For more information about GTL see [9].

\section{FREEWAY TRAFFIC MODEL}

In this paper, we use the Cell Transmission Model (CTM) [10] as a mathematical representation of the freeway traffic. CTM can be seen as Godunov's difference scheme [11] under the assumption that the density-flow relation (fundamental diagram) is given in triangular form.

A freeway is divided into cells as depicted in Fig. 1. Each section is assumed to be equipped with at most one on-ramp 
and one off-ramp. For a section $i$ we assume the following notation: $\rho_{i}$ - vehicle density, $\phi_{i}$ - mainstream flow entering a section, $u_{i}$ - on-ramp demand, $r_{i}$ - on-ramp flow entering a section, $s_{i}$ - off-ramp flow leaving a section, $v_{i}$ - free flow velocity, $w_{i}$ - congestion wave speed, $F_{i}$ - capacity, $\bar{\rho}_{i}-$ jam density, $l_{i}-$ section length.

\section{A. Governing equation}

According to CTM, the evolution of the system, for a section $i$ and time instant $k+1$, is described by the difference equation:

$$
\rho_{i}(k+1)=\rho_{i}(k)+\frac{\Delta t}{l_{i}}\left[\Phi_{i}^{+}(k)-\Phi_{i}^{-}(k)\right],
$$

where the initial state $\rho(k=0)$ is given. The time step $\Delta t$ between instants $k$ and $k+1$ must be taken small enough to fulfil the convergence condition (for details see [12]). $\Phi_{i}^{+}(k)$ and $\Phi_{i}^{-}(k)$ stands for the total flow entering and leaving a section $i$, respectively, i.e.:

$$
\Phi_{i}^{+}(k)=\phi_{i}(k)+r_{i}(k), \Phi_{i}^{-}(k)=\phi_{i+1}(k)+s_{i}(k) .
$$

Before we give the explicit formulas for $\phi, r$ and $s$, we recall a few facts on the existing models for merging and diverging of traffic flows. Unlike in the case of diverging, where the widely accepted is the hypothesis that the flow entering the off-ramp is a portion of the total flow leaving the section, merging phenomenon is much more difficult to describe. Several merge models have been introduced and used for ramp metering design. Some of them are oversimplified (see for example [13], [14]) and can produce unrealistic situations, where for instance the sum of upstream flows is greater than downstream supply. On the other hand, too sophisticated models, which for example take into account the dynamics of cars entering a mainline [15], are also much more challenging for tuning and applying to optimal control. In this paper, we use Daganzo's Priority Merge Model [16] that seems to be a good compromise between accuracy and complexity. The model consist of so called merging parameter $p$ that captures the priority between mainstream flow $\phi$ and on-ramp flow $r$ when merging in a section during the congestion. In order to specify the merging parameter, one should consider geometric properties of on-ramp as well as drivers' behavior. The detailed study will be presented in the section V-B.

By introducing Demand $D_{i}$ and Supply $S_{i}$ functions:

$$
\begin{aligned}
& D_{i}(k)=\min \left\{\bar{\beta}_{i} v_{i} \rho_{i}(k), F_{i}\right\}, \\
& S_{i}(k)=\min \left\{w_{i}\left(\bar{\rho}_{i}-\rho_{i}(k)\right), F_{i}\right\}
\end{aligned}
$$

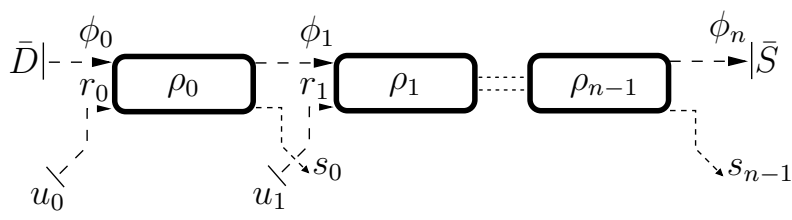

Fig. 1. Freeway divided into n sections. Each section is accompanied with at most one on-ramp and one off-ramp. the mainstream and on-ramp flows can be computed as follows:

$$
\begin{aligned}
& \text { if } D_{i-1}(k)+u_{i}(k) \leq S_{i}(k): \\
& \phi_{i}(k)=D_{i-1}(k), r_{i}(k)=u_{i}(k) \\
& \text { otherwise : } \\
& \phi_{i}(k)=\operatorname{mid}\left\{D_{i-1}(k), S_{i}(k)-u_{i}(k),\left(1-p_{i}\right) S_{i}(k)\right\}, \\
& r_{i}(k)=\operatorname{mid}\left\{u_{i}(k), S_{i}(k)-D_{i-1}(k), p_{i} S_{i}(k)\right\} .
\end{aligned}
$$

Here the function $\operatorname{mid}\{\cdot\}$ returns the middle value, i.e. for example mid $\{a, b, c\}=a$ if $b \leq a \leq c$. For the off-ramp flows we assume:

$$
s_{i}(k)=\frac{1-\bar{\beta}_{i}}{\bar{\beta}_{i}} \phi_{i+1}(k) .
$$

The parameter $\bar{\beta}_{i}=1-\beta_{i}$, where $\beta_{i} \in[0,1)$, stands for the split ratio. The boundary conditions are determined by some demand $\bar{D}(k)=D_{-1}(k)$ for the section 0 and some supply $\bar{S}(k)=S_{n}(k)$ for the section $n-1$. For the flow $\phi_{n}$, under the absence of on-ramp, we can simply write:

$$
\phi_{n}(k)=\min \left\{D_{n-1}(k), \bar{S}(k)\right\} .
$$

For the convenience of further study we will rewrite the governing equation in more compact form. By introducing the state vector $x \in \mathcal{X}$ and the input vector $u \in \mathcal{U}$ that stand for vehicle densities and on-ramp demands, respectively, we can represent the governing equation in a form of a switching control system:

$$
\begin{aligned}
& x(k+1)=A_{s(k)} x(k)+B_{s(k)} u(k)+C_{s(k)}(k), \\
& s(k)=f(x(k), u(k)) .
\end{aligned}
$$

Here $x=\left(\rho_{0}, \rho_{1}, \ldots, \rho_{n-1}\right), u=\left(u_{0}, u_{1}, \ldots, u_{n-1}\right)$. The switching variable $s \in \mathcal{S}=\{1,2, \ldots, m\}$ indicates a mode of the system, and it determines components for the matrices $A^{n \times n}, B^{n \times n}$ and for the vector $C^{n \times 1}(k)$. Notice that the total number of all possible modes, generated by the formulas for mainstream and on-ramp flows, is equal to $8^{n}$, and, as it will be shown later, this is the crucial fact for the way the optimization is performed. The boundary functions $\bar{D}(k)$ and $\bar{S}(k)$ appear in the vector $C^{n \times 1}(k)$.

\section{OPTIMAL BALANCING OF STEADY STATES}

In this section, we recall in brief the study on optimization problems that we presented in the paper [2]. Let us consider the set of steady states of a freeway system represented by (7). The goal was to find the inputs $u$ such that the corresponding steady state is balanced, i.e. the values of the state vector $x$ are equalized. As it was shown in [1], for the existence of the balanced steady states some strong conditions imposed on the cell parameters must hold. Thus, instead of exactly balanced steady state we tried to design the one (here called non-exact balanced) that stays as close as possible to some desired exact balance. For this purpose we posed the optimization problem, where the goal was to minimize the relevant norm corresponding to the distance between this desired exact balance and the set of steady 
states. Regarding the fact that we consider the switching system with a large number of switching modes, we proposed a convex formulation that allowed us to obtain the solution in a reasonable time. For a desired exactly balanced steady state, denoted here by $c^{*} \mathbf{1}$ (here $\mathbf{1}$ is all-ones vector), we assumed the one that maximizes the Total Travel Distance (TTD).

At first, we consider the problem of finding the value for $c^{*}$. Under the assumption that the system is in the steady state, for the total length of a freeway and for some time interval $T$, the Total Travel Distance can be written as follows:

$$
\mathrm{TTD}=T \sum_{i=0}^{n-1} \min \left\{v_{i} \rho_{i}, w_{i}\left(\bar{\rho}_{i}-\rho_{i}\right)\right\} l_{i} .
$$

Note that the expression $\min \left\{v_{i} \rho_{i}, w_{i}\left(\bar{\rho}_{i}-\rho_{i}\right)\right\}$ is for the average flow inside a section $i$. Thus, we can consider the following problem:

$$
\begin{aligned}
\text { Find } c^{*} & =\operatorname{argmax}_{c} J_{1}(c), \\
J_{1}(c) & =\sum_{i=0}^{n-1} \min \left\{v_{i} c, w_{i}\left(\bar{\rho}_{i}-c\right)\right\} l_{i} .
\end{aligned}
$$

The second step is to find the input vector $u^{*}$ such that the corresponding steady state $x^{*}$ is the closest to $c^{*} \mathbf{1}$ when considering the quadratic norm. As it was shown in [2], the set of equilibrium points for (7) can be described by the set of systems of linear equations and inequalities. The problem can be stated as follows:

$$
\begin{aligned}
& \text { Find }\left(x^{*}, u^{*}\right)=\operatorname{argmin}_{x \in \mathcal{X}, u \in \mathcal{U}} J_{2}(x), \\
& \qquad \begin{array}{c}
J_{2}(x)=\left(x-c^{*} \mathbf{1}\right)^{T} Q_{1}\left(x-c^{*} \mathbf{1}\right)+\gamma x^{T} Q_{2} x \\
\text { under } \quad\left(A_{s}-\mathbf{I}\right) x+B_{s} u+C_{s}=0, \\
K_{s} x+L_{s} u+M_{s} \leq 0, s \in \mathcal{S}
\end{array}
\end{aligned}
$$

Here, $\mathcal{S}$ is the set of these modes for which there exists a solution and it is unique for $x^{*}$. In other words, we discard the modes such that $\left(A_{s}-\mathbf{I}\right)$ is singular or $B_{s}$ is null matrix. The first term in the objective function corresponds to the distance of the steady state vector from $c^{*} \mathbf{1}$ while the second one measures the accuracy of the balance and it is introduced to minimize the distance between the components of the state vector. $Q_{1}$ is assumed to be positive-defined symmetrical and it weights the priorities for the on-ramps. $Q_{2}$ is the Laplacian matrix that is given in the form:

$$
Q_{2}(i, j)= \begin{cases}n-1 & \text { if } i=j \\ -1 & \text { otherwise }\end{cases}
$$

The objective terms are weighted by specifying $\gamma$.

\section{NUMERICAL IMPLEMENTATION}

In order to solve the optimization problems presented in the previous section we developed and implemented relevant numerical procedures. The solution methods were already presented in details in the paper [2]. Here we discuss the implementation aspects highlighting the difficulties and limitations in size of tractable problem. This size will correspond here to the number of freeway cells $(n)$.

The first optimization problem (9) was reformulated into a set of linear constrained problems. The algorithm presented in the paper [2] uses some facts about the signs of terms that appear into linear constraints. Thanks to this, it is very easy for coding and it allows us to solve the problem of the size $n=15$ in less than one second and $n=20$ in less than one minute. Notice that the total number of linear sub-problems to be solved is equal to $2^{n}$.

Unlike in the case of the problem (9), where under the assumption that for a certain time the cell parameters remain constant and the computation can be performed off-line, the second optimization problem (10) is supposed to be updated every reasonable time period, as the boundary conditions change. Therefore, remembering the fact that the number of sub-problems to be solved is related to huge number $8^{n}$ (total number of all possible modes), we need to investigate carefully the computational capabilities of the software we built.

The codes consists of two major parts. The first part is to introduce the constraints, and therefore to create the matrices $A, B, K, L$ and the vectors $C, M$ for all considered modes of the system. For that purpose, we introduced relevant combinatorial procedures that allowed us to generate the full set of matrices for all possible modes. At this level we also discard the modes that do not meet the previously mentioned conditions for existence and uniqueness of solutions. The second part is to solve the remained optimization subproblems. These are formulated as the convex quadratic problems so that efficient solvers can be applied. In our programs we used Matlab quadprog function. The computations for different number of freeway cells were performed by using a personal computer with Core 2 Duo processor $(1.4 \mathrm{GHz}, 2 \mathrm{~GB}$ RAM). The computational times are summarized in the Table

TABLE I

COMPUTATIONAL TIME FOR THE PROBLEM (10) WITH RESPECT TO THE NUMBER OF FREEWAY CELLS.

\begin{tabular}{|c|c|c|}
\hline nr. of cells & nr. of sub-problems & computational time [s] \\
\hline 4 & 185 & 1 \\
\hline 5 & 853 & 3 \\
\hline 6 & 3820 & 11 \\
\hline 7 & 16829 & 55 \\
\hline 8 & 73439 & 260 \\
\hline
\end{tabular}

I. Assuming that the computations need to be repeated not less than every one or two minutes, the size of the system should be at most $n=7$. This is a significant limitation. However, in case of a freeway where the parameters are almost uniformly distributed, we can use a model with a reduced number of cells and then the proposed method can be applied to the length of several kilometers.

\section{CALIBRATION OF THE MODEL}

In this section, we tune the CTM model to represent the Grenoble South Ring. To do this, we use real demand 
information collected from the ring and then calibrate the model parameters by running Aimsun micro-simulator (see [18]). For the optimization we choose a part of the ring that contains 4 on-ramps and 4 off-ramps.

\section{A. CTM representation of the Grenoble South Ring}

The Grenoble South Ring is of total length 10.5 [km] equipped with 11 on-ramps and 10 off-ramps. It connects the city of Grenoble in the north-east to south-west linking the highways A41 and A480.

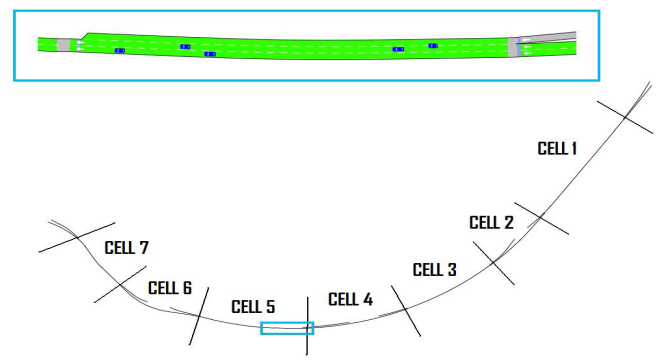

Fig. 2. Aimsun representation of the Grenoble South Ring and the cell division used in CTM.

In order to build CTM representation, the following steps were performed: 24 hours real data collection for the on-ramp and boundary flows, architecture design in Aimsun, split ratio estimation by using the real data and Aimsun microsimulator, cell division, 24 hours Aimsun micro-simulations and collection of data from virtual sensors placed along the ring, data processing and estimation of the cell parameters $(v, w, \bar{\rho})$.

In this paper, we consider a part of the Grenoble South Ring of length $4.7[\mathrm{~km}]$ including 4 on-ramps and 4 offramps as depicted in Fig. 2. Respecting the computational limitations, mentioned in the previous section, the freeway was divided into 7 cells. The estimated parameters are summarized in the Table II. Only the cells with the odd numbers (indicated by ${ }^{*}$ ) are equipped with one on-ramp and one off-ramp.

\section{B. Merging Parameter Calibration}

The key point for the best performance of ramp metering strategies is a proper calibration of the merging model parameter $p$. For that purpose, we made several experiments by using the Aimsun micro-simulator. The goal was to

TABLE II

CTM PARAMETERS FOR THE GRENOBLE SOUTH RING.

\begin{tabular}{|c|c|c|c|c|}
\hline cell nr. & $L[\mathrm{~km}]$ & $v[\mathrm{~km} / \mathrm{h}]$ & $w[\mathrm{~km} / \mathrm{h}]$ & $\bar{\rho}[\mathrm{veh} / \mathrm{km}]$ \\
\hline $1^{*}$ & 0.96 & 70 & 15 & 445 \\
\hline 2 & 0.51 & 73 & 18 & 412 \\
\hline $3^{*}$ & 0.59 & 70 & 16 & 428 \\
\hline 4 & 0.65 & 71 & 18 & 407 \\
\hline $5^{*}$ & 0.64 & 70 & 19 & 407 \\
\hline 6 & 0.56 & 75 & 18 & 412 \\
\hline $7^{*}$ & 0.80 & 71 & 19 & 425 \\
\hline
\end{tabular}

determine the interval in which the merging parameter may vary when taking into account various drivers' behavior.

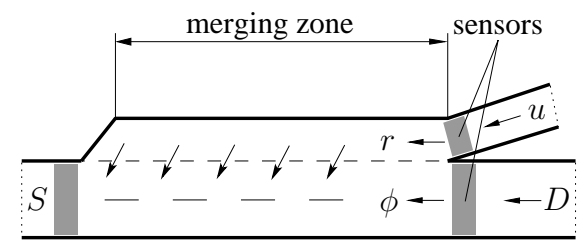

Fig. 3. On-ramp and mainstream flow merging experiment: sensors placement.

Let us consider the on-ramp as depicted in Fig. 3. According to the model (4), in the case of large mainstream and on-ramp demands, i.e. satisfying $D+u>S, D \geq(1-p) S$, $u \geq p S$, the entering flows are $\phi=(1-p) S$ and $r=p S$. Therefore, in such a case $p$ can be simply determined by measuring the flows $r$ and $\phi$, then using $p=r /(\phi+r)$.

In the simulations, we created a downstream bottleneck such that the supply was $S \approx 2200$ [veh/h]. For the demands we set $D=2200[\mathrm{veh} / \mathrm{h}]$ and $u=1100[\mathrm{veh} / \mathrm{h}]$. The simulations were performed for two hours. During the second hour, when we observed almost steady state, the entering flows were measured by the virtual sensors as shown in Fig. 4 (the blue belts). We considered five different lengths of merging zone (60-140 meters). The drivers' behavior at the Aimsun micro-simulator was changed by setting the lane changing cooperation parameter which may vary from $0 \%$ to $100 \%$. $0 \%$ corresponds to extremely egotistic driving, where on-ramp queue enters mainstream very slowly. By increasing the parameter we can observe more driver's cooperation and the will to change the lanes so to enable the on-ramp vehicles join a freeway. The simulation results are presented in the Table III. As we may expect, the lane changing cooperation

TABLE III

THE MERGING PARAMETER ( $p$ ) VALUES WITH RESPECT TO DIFFERENT LENGTH OF THE MERGING ZONE AND VARIOUS DRIVERS' BEHAVIOR.

\begin{tabular}{|c|c|c|c|c|c|}
\hline Merging zone & \multicolumn{5}{|c|}{ Lane changing cooperation } \\
\cline { 2 - 6 } length [m] & $5 \%$ & $25 \%$ & $50 \%$ & $75 \%$ & $100 \%$ \\
\hline 60 & 0.096 & 0.119 & 0.170 & 0.213 & 0.251 \\
\hline 80 & 0.100 & 0.120 & 0.164 & 0.214 & 0.250 \\
\hline 100 & 0.093 & 0.115 & 0.163 & 0.217 & 0.251 \\
\hline 120 & 0.119 & 0.125 & 0.189 & 0.231 & 0.251 \\
\hline 140 & 0.121 & 0.151 & 0.207 & 0.241 & 0.255 \\
\hline
\end{tabular}

parameter strongly determines the merging priorities. The relation between $p$ and the lane changing cooperation parameter is not linear, but it is clearly increasing. Surprising

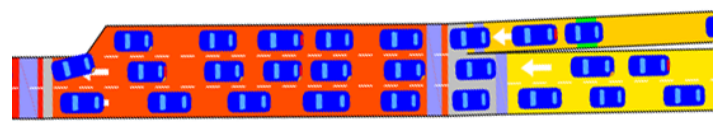

Fig. 4. The merging flow experiment performed with the Aimsun microsimulator 
is that in some cases $p$ decreases when the merging zone becomes longer. The important observation is that for $100 \%$ of cooperation there exists a saturation point where $p \approx$ 0.25 . This can be intuitively explained as follows. Among two mainstream lanes the left one is taken by the vehicles travelling from upstream while the right one is to be shared by the upstream and the on-ramp demands with the same priority. Therefore, the on-ramp flow is approximately equal to $1 / 4$ of the total downstream supply.

For the model of the Grenoble South Ring we assume the following merging parameter values: $p_{1}=0.2, p_{3}=0.18$, $p_{5}=0.21, p_{7}=0.17$.

\section{STEADY STATE BALANCING ON THE GRENOBLE SOUTH RING: CASE STUDIES}

In this section, we discuss the solutions of the optimization problems (9), (10). We begin with two simple examples to show the condition under which the design of the exact balance may be feasible. Next, we consider different traffic scenarios on south-ring in Grenoble. For every computation, for the objective function $J_{2}$ we assume that $Q_{1}$ is the Identity matrix and the weighting parameter $\gamma$ is equal to 0.1 .

\section{A. Exact and non-exact balance}

Let us consider a freeway given with the following set of the cell parameters: $v=[80,80,85,85,90,90,95][\mathrm{km} / \mathrm{h}]$, $w_{i}=25[\mathrm{~km} / \mathrm{h}], \bar{\rho}_{i}=400[\mathrm{veh} / \mathrm{km}], \bar{\beta}_{i}=1$, for $i=$ $1,2, \ldots, 7$ and $p_{i}=0.2$ for $i=1,3,5,7$. For the boundary conditions we assume $\bar{D}=3000[\mathrm{veh} / \mathrm{h}]$ and $\bar{S}=7000$ [veh/h]. The bounds for the inputs are set as follows: $u_{i}^{\min }=$ 0 and $u_{i}^{\max }=3000[\mathrm{veh} / \mathrm{h}]$ for $i=1,3,5,7$. We assume that the desired exact balance is $c^{*}=70[\mathrm{veh} / \mathrm{km}]$. The solution of the optimization problem (10) is presented in the Table IV. This is an example of the exact balance $\left(J_{2}=0\right)$. It was

TABLE IV

INPUT AND THE CORRESPONDING STEADY STATE IN THE CASE OF THE EXACT BALANCE.

\begin{tabular}{|c|c|c|c|c|c|c|c|}
\hline Cell nr. & 1 & 2 & 3 & 4 & 5 & 6 & 7 \\
\hline$u^{*}[\mathrm{veh} / \mathrm{h}]$ & 2600 & - & 350 & - & 350 & - & 350 \\
\hline$x^{*}[\mathrm{veh} / \mathrm{km}]$ & 70 & 70 & 70 & 70 & 70 & 70 & 70 \\
\hline
\end{tabular}

proven in [1] that in the case where all cell are in the free flow state free state (i.e. $v_{i} x_{i} \leq F_{i}$ for $i=0,1, \ldots, n-1$ ), the exactly balanced steady state is feasible only if:

$$
\bar{\beta}_{i-1} v_{i-1} \leq v_{i}, \quad i=1,2, \ldots, n-1 .
$$

In the following example we show the solution of (10), when the condition (12) is not fulfilled. We consider the same set of parameters except that we reverse values in the vector of free flow velocity, i.e. we set: $v=[95,90,90,85,85,80,80]$. In such a case, the optimal solution is clearly unbalanced (see the Table $\mathrm{V}$ and Fig. 5) with the objective function $J_{2}=$ $0.2 \cdot 10^{3}$.
TABLE V

OPTIMAL INPUT AND THE CORRESPONDING STEADY STATE IN THE CASE OF THE NON-EXACT BALANCE.

\begin{tabular}{|c|c|c|c|c|c|c|c|}
\hline Cell nr. & 1 & 2 & 3 & 4 & 5 & 6 & 7 \\
\hline$u^{*}[\mathrm{veh} / \mathrm{h}]$ & 2993 & - & 0 & - & 0 & - & 0 \\
\hline$x^{*}[\mathrm{veh} / \mathrm{km}]$ & 63 & 66.5 & 66.5 & 70.5 & 70.5 & 74.9 & 74.9 \\
\hline
\end{tabular}

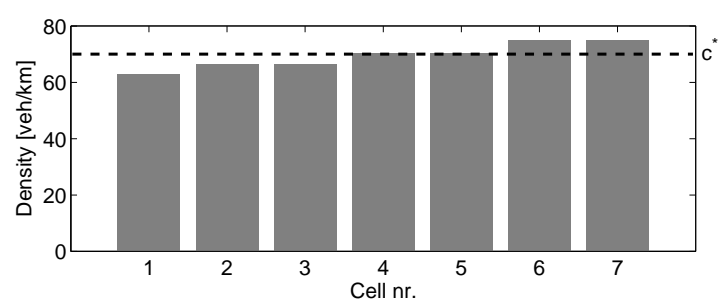

Fig. 5. Steady state traffic density distribution: the case of the non-exact balance.

\section{B. The Grenoble South Ring scenarios}

In this section, we work on previously estimated model parameters as it was shown in the Table II. In this case, the solution of the problem (9) is $c^{*}=86.8[\mathrm{veh} / \mathrm{km}]$. The corresponding Total Travel Distance for some time period $T$ is $T T D=T \cdot 2.73 \cdot 10^{4}[\mathrm{veh} \cdot \mathrm{km}]$. We assume the traffic scenario where the boundary conditions and the split ratios are given as follows: $\bar{D}=3000[\mathrm{veh} / \mathrm{h}], \bar{S}=7000$ [veh/h], $\bar{\beta}_{i}=0.9$ for $i=1,3,5,7$. We set the following minimum and maximum value for each of the inputs (onramp demand): $u^{\min }=0, u^{\max }=2000[\mathrm{veh} / \mathrm{h}]$. The optimal solution of the problem (9) is presented in the Table VI. The corresponding value for the objective function is $J_{2}=1.37 \cdot 10^{3}$.

TABLE VI

OPTIMAL INPUT AND THE CORRESPONDING STEADY STATE.

\begin{tabular}{|c|c|c|c|c|c|c|c|}
\hline Cell nr. & 1 & 2 & 3 & 4 & 5 & 6 & 7 \\
\hline$u^{*}[\mathrm{veh} / \mathrm{h}]$ & 1724 & - & 1073 & - & 1064 & - & 631 \\
\hline$x^{*}[\mathrm{veh} / \mathrm{km}]$ & 67.5 & 58.3 & 76.1 & 67.5 & 83.7 & 70.3 & 83.1 \\
\hline
\end{tabular}

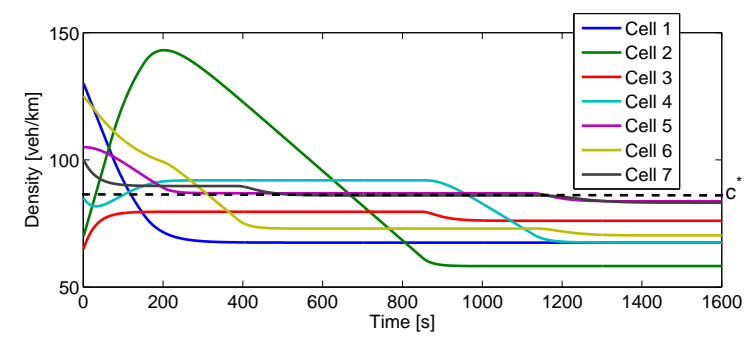

Fig. 6. The system dynamics under the optimal inputs and the fixed boundary and split ratio conditions.

Fig. 6 presents the evolution of the system from the initial point $x_{i n i}=[130,70,65,85,105,125,100]$. The convergence time depends on the modes that the system must go 


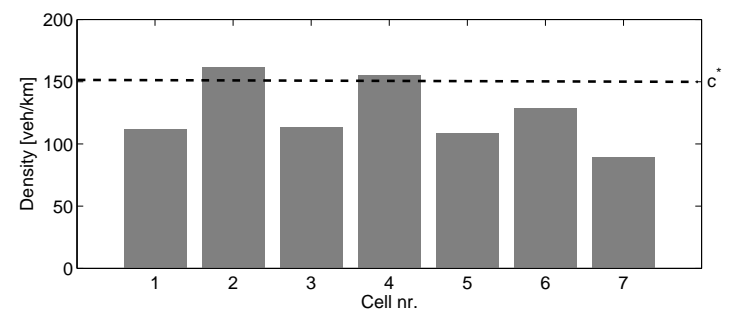

Fig. 7. Steady state traffic density distribution: the case of a high external demand.

through to approach a steady state. In most of the cases, this time is less than several minutes. It becomes significantly longer, if a desired steady state is very close to the boundary that triggers mode switching.

In the next example, we consider the situation under a high external demand. The goal is to avoid long queueing time, and therefore we accept to keep higher density on the highway. We assume $c^{*}=150[\mathrm{veh} / \mathrm{km}]$. The optimal input and the corresponding steady state are presented in the Table VII (see also Fig. 7). In case of large $c^{*}$, the solution becomes more unbalanced and more distant from $c^{*} \mathbf{1}$. This is due to the fact that increase of vehicle density results in a higher sparsity of the matrices $B_{s}$, and therefore the system becomes more underactuated.

The Table VIII shows how the objective function and thus the balance accuracy is affected by different boundary conditions. The computations were performed for $c^{*}=$ 86.8. The boundary conditions as well as all of the cell parameters influence the shape of the set of equilibrium points. Intuitively we may expect that larger the difference $\bar{S}-\bar{D}$ then larger the equilibrium set, and therefore it is easier to find inside this set a desired steady state. At this point we need to notice that the equilibrium set is also determined by the input bounds, and as the results show, in some cases it is more convenient to have larger boundary demand and smaller boundary supply.

\section{CONCLUSIONS AND FUTURE WORKS}

The paper captures several aspects of the implementation of the idea of optimal balancing of freeway traffic density. The significant limitation is the size of the tractable optimization problem that is to be solved every time period that depends on how fast the traffic demands are changing. The proposed method allows us to solve the problem in less than one minute if the number of cells in CTM does not exceed seven. In case of the Grenoble South Ring it allows

TABLE VII

OPTIMAL INPUT AND THE CORRESPONDING STEADY STATE IN THE CASE OF A HIGH EXTERNAL DEMAND.

\begin{tabular}{|c|c|c|c|c|c|c|c|}
\hline Cell nr. & 1 & 2 & 3 & 4 & 5 & 6 & 7 \\
\hline$u^{*}[\mathrm{veh} / \mathrm{h}]$ & 2000 & - & 531 & - & 2000 & - & 1274 \\
\hline$x^{*}[\mathrm{veh} / \mathrm{km}]$ & 111.6 & 162 & 113.5 & 155.3 & 109 & 128.8 & 89.7 \\
\hline
\end{tabular}

TABLE VIII

OBJECTIVE FUNCTION VALUES WITH RESPECT TO DIFFERENT BOUNDARY CONDITIONS.

\begin{tabular}{|c|c|c|c|c|c|}
\hline $\bar{D}[\mathrm{veh} / \mathrm{h}]$ & 2000 & 4000 & 6000 & 4000 & 5000 \\
\hline$S[\mathrm{veh} / \mathrm{h}]$ & 7000 & 7000 & 7000 & 5000 & 4000 \\
\hline$J_{2} / 10^{3}$ & 4.25 & 3.33 & 1.68 & 1.35 & 2.85 \\
\hline
\end{tabular}

us to work on the length of 4.7 kilometers. This length can be potentially expanded, if we introduce the additional procedures to solve the optimization problem which takes into account only the closest modes to the one that the system is currently operating on. The on-going topic of the authors is to design a distributed optimal feedback controller that keeps the system on a desired near balanced state.

\section{REFERENCES}

[1] D. Pisarski, C. Canudas-de-Wit, Analysis and Design of Equilibrium Points for the Cell-Transmission Traffic Model, IEEE American Control Conference, 2012, pp 5763-5768.

[2] D. Pisarski, C. Canudas-de-Wit, Optimal Balancing of Road Traffic Density Distributions for the Cell Transmission Model, IEEE Conference on Decision and Control, 2012, pp 6969-6974.

[3] G. Gomes, R. Horowitz, Optimal Freeway Ramp Metering Using the Asymmetric Cell Transmission Model, Transportation Research Part C, vol. 14, 2006, pp 244-262.

[4] A. Hegyi, B. De Schutter, J. Hellendroon, T. van den Boom, Optimal Coordination of Ramp Metering and Variable Speed Control - An MPC Approach, IEEE American Control Conference, 2002, pp 36003605.

[5] A. Alessandri, A. Di Febbraro, A. Ferrara, E. Punta. Optimal Control of Freeways via Speed Signalling and Ramp Metering, Control Engineering Practice, vol. 6, 1998, pp 771-780.

[6] A. Di Febbraro, T. Parisini, S. Sacone, R. Zoppoli, Neural Approximation for Feedback Optimal Control of Freeway Systems, IEEE Transactions on Vehicular Technology, vol. 50(1), 2001, pp 302-312.

[7] C. Canudas De Wit, L. Leon, A. Kibangou: Graph constrained-CTM observer design for the Grenoble south ring, 13-th IFAC Symposium on Control in Transportation Systems, 2012.

[8] L. Leon, C. Canudas De Wit, A. Kibangou: Adaptive Kalman Filtering for Multi-Step ahead Traffic Flow Prediction, IEEE American Control Conference, 2013.

[9] Grenoble Traffic Laboratory: http://necs.inrialpes.fr/gtl/index.html

[10] C. Daganzo, The Cell Transmission Model: A Dynamic Representation of Highway Traffic Consistent with the Hydrodynamic Theory, Transportation Research Part B, vol. 28, 1994, pp 269-287.

[11] S. K. Godunov, A Difference Scheme for Numerical Solution of Discontinuous Solution of Hydrodynamic Equations, Math. Sbornik, vol 47, 271âĂŞ306, translated US Joint Publ. Res. Service, JPRS 7226, 1969.

[12] R. J. LeVeque, Numerical Methods for Conservation Laws, Birkhauser Verlag, Basel; 1992.

[13] J. P. Lebacque, The Godunov Scheme and What It Means for First Order Traffic Flows Models, International Symposium on Transportation and Traffic Theory, vol. 13, 1996, pp 647-677.

[14] W. L. Jin, H. M. Zhang, On the Distribution Schemes for Determining Flows Through a Merge, Transportation Research Part B, vol. 37, 2003, pp 521-540.

[15] L. Leclercq, J. A. Laval, N. Chiabaut, Capacity Drops at Merges: An Endogenous Model, International Symposium on Transportation and Traffic Theory, vol. 17, 2011, pp 12-26.

[16] C. Daganzo, The Cell Transmission Model: Part II: Network Traffic, Transportation Research Part B, vol. 29, 1995, pp 79-93.

[17] G. F. Newell, A simplified Theory of Kinematic Waves in Highway Traffic, Part I: General theory, Transportation Research Part B, vol 27, 1993, pp 281âĂŞ287.

[18] TSS-Transport Simulation Systems: http://www.aimsun.com 